MS21 Intermolecular interactions from structural, energetic and charge density perspective(s)

Chairs: Prof. Enrique Espinosa, Prof. Ulli Englert

MS21-01

\section{Stay hydrated - where pharmaceutical hydrates come from and why}

Katharina Edkins ${ }^{1}$

1. School of Pharmacy, Queen's University Belfast, Belfast, United Kingdom

email: k.edkins@qub.ac.uk

Multiple crystal forms in pharmaceuticals pose severe risks to the formulation of a novel medicine, and to its safety and efficacy. In particular, the inclusion of water into the crystal lattice and thus the formation of crystalline hydrates generates challenging materials for formulation due to their thermodynamic low water solubility. Variations in hydrate stability in addition to the existence of stoichiometric and non-stoichiometric behaviour pose additional problems, which require non-trivial solutions. It is thus a major area of interest in the pharmaceutical industry to discover hydrated crystal forms and avoid them if possible.

The classic approach of hydrate research is the solvent screening of a compound to explore the crystallisation space and detect as many crystal forms as experimentally accessible, which are then analysed in detail. ${ }^{1,2}$ Whilst this approach gives detailed insight into the stability of the chosen crystal form and can explain their solid-state behaviour, it is a retrospect approach in which the hydrate must be found before it can be analysed. We are interested in interactions being present in solution before crystallisation and their connection to the resulting crystal form with the aim to predict hydrate formation before conducting the crystallisation experiment. The use of spectroscopic methods in combination with neutron scattering and simulation proves particularly powerful, and this approach will be presented using several well-known drug compounds ranging in their hydrate stability and water solubility.

Keywords: Hydrogen bonding, crystal form, pre-nucleation aggregates

\section{MS21-02}

\section{Chemical insights from systematic studies of interactions in re...}

Simon Coles ${ }^{1}$

1. Chemistry, University of Southampton, Southampton, United Kingdom

email: s.j.coles@soton.ac.uk

Many industries, most notably pharmaceutical, have for some time been using compound libraries as a systematic approach to comprehensively understanding a chemical landscape. Screening in medicinal chemistry is an obvious example and QSAR takes a similar, in-silico, approach. Understanding packing and structure-determining factors in the solid state is key in many areas eg polymorphism or crystallization, and of course we need to understand this behaviour if we are to control solid-state formation in any way. Our research uses systematic studies on the crystal structures of homologous series of compounds to derive rules and begin to predict solid-state behaviour. We now extend this concept to the application of Quantum Crystallography approaches to series of compounds in order to further understanding of structure and properties.

The talk will present examples of series of compounds specifically designed to examine:

1. Transition states for $\mathrm{N}-\mathrm{C}$ bond formation;

2. Tuning receptor structure to optimise anion binding;

3. Co-former selection for pharmaceutical co-crystals;

4. Rationalising properties of co-crystals;

5. The role of fluorine interactions in the organic solid state;

6. Tailoring aromatic intermolecular interactions to activate spin-crossover materials.

In all of the above, a deep understanding of the complementary and competitive energetic contributions is necessary in order to rationalise solid-state structures and their behaviour.

Keywords: Quantum Crystallography, Structural Systematics 